\title{
Apresentando o TratBem: Suporte Social, Informacional e Psicológico ao Tabagista
}

\author{
Francisco Oliveira ${ }^{1}$, Maria da Penha Uchoa Sales ${ }^{2}$, Joyce Horn Fonteles ${ }^{3}$, Nayara \\ Nogueira Silva ${ }^{1}$, Felipe Chagas Pereira ${ }^{1}$, Janaína Farias de Melo ${ }^{4}$, Isabele \\ Assunção Mendonça Cavalcante ${ }^{4}$, Ligia de Moraes $^{3}$ \\ 1Universidade Estadual do Ceará - Fortaleza - CE - Brasil \\ ${ }^{2}$ Hospital de Messejana Dr. Carlos Alberto Studart - Fortaleza - CE - Brasil \\ ${ }^{3}$ Universidade de Fortaleza - Fortaleza - CE - Brasil. \\ ${ }^{4}$ Universidade Federal do Ceará - Fortaleza - CE - Brasil. \\ \{fran.mb.oliveira, puchoasales152, hornjoyce, \\ nayara.nogueiral, felip.uece\}@gmail.com, \\ janjanmelo@uol.com.br, \{isabeleamc, \\ liginhademoraes\} @hotmail.com
}

\begin{abstract}
Smoking is the number one preventable cause of death in the world. Only 5\% of those who try to quit without support succeed. The best support available today in Brazil can be found anti-tobacco groups hosted in public hospitals, which can help only a few patients at a time. We introduce TratBem, a computational system capable of delivering informational, social and psychological support anytime anywhere to smokers. We present a feasibility study for the use of mobile computing in public healthcare, followed by another study of how the introduction of TratBem impacted the lives of the smokers and raised their abstinence rates.
\end{abstract}

Resumo. O tabagismo é a maior causa de morte evitável no mundo. Apenas $5 \%$ daqueles que tentam parar de fumar sem apoio são bem sucedidos. $O$ melhor apoio disponível no Brasil encontra-se nos grupos antitabagismo dos hospitais públicos, que possuem vagas limitadas. Apresenta-se o TratBem, um sistema computacional capaz de promover suporte informacional, social $e$ psicológico a fumantes a qualquer hora em qualquer lugar. Discute-se também um estudo de viabilidade do uso de computação móvel em saúde pública, seguido de outro estudo como a introdução do TratBem aumentou as taxas de abstinência.

\section{Introdução}

O tabagismo é a maior causa de morte evitável no mundo, cujo número anual é de cerca de 5 milhões (que equivale a uma morte a cada 6 segundos) [WHO 2011]. Estima-se que no Brasil a adicção mate duzentas mil pessoas ao ano [Ministério da Saúde 2013]. O Governo Federal Brasileiro gastou em 2012, cerca de R $\$ 60$ milhões somente com medicamentos em tratamentos [Ministério da Saúde 2013]. Ainda, segundo a mesma fonte, $14,8 \%$ dos brasileiros com mais de 18 anos fumam. O tabagismo provoca $70 \%$ dos cânceres de pulmão, $42 \%$ das doenças respiratórias crônicas e $10 \%$ das doenças do 
aparelho respiratório [Mathers, Stevens \& Mascarenhas 2009]. Pesquisadores alertam que, para reverter esses números, é preciso intervenções que atinjam milhões de pessoas de forma amigável [Cobb, Graham, Byron, Niaura, Abrams et al. 2011].

As estratégias de ataque ao problema empregadas pelos governos são extremadas. Existem campanhas de marketing que atingem milhões de pessoas, com excelente alcance, porém, por ser impessoal, é uma estratégia pouco eficaz. Por outro lado, há centros de apoio ao tabagista. Nessa abordagem, a eficácia é máxima, principalmente quando se tratam de grupos com a participação de profissionais de saúde. Nesses, os pacientes recebem diversos tipos de suporte: 1) o de profissionais de saúde propriamente dito; 2) o social, obtido através do convívio com outros que possuem a mesma demanda e 3) o medicamentoso, em que recebem gratuitamente a medicação prescrita (no caso de centros públicos). Esses diversos tipos de apoio fazem com que a eficácia da intervenção seja bastante elevada. Contudo, o alcance obtido ainda é considerado baixo, pois: 1) O número de vagas nesses centros é sempre muito inferior à demanda; 2) os serviços são normalmente oferecidos em horário comercial, em que a maioria das pessoas está trabalhando; e 3) diversos pacientes não se sentem motivados a entrar nas filas de espera para esse tipo de atendimento.

As tecnologias de informação e comunicação (TICs) oferecem o potencial de combinar as vantagens dos tratamentos intensivos (que são personalizados) com as intervenções de longo alcance, através da incorporação de respostas interativas, adaptando estratégias de contato pessoal [Michael \& Cheuvront 1998]. Pesquisas destacam o alto potencial das redes sociais na promoção da adoção de comportamentos mais saudáveis [Cobb, Graham, Abrams et al. 2010]. Intervenções de rede sociais podem funcionar através de diversos mecanismos, incluindo influência e suporte social, bem como transferência de informação [Cobb et al. 2010]. Redes sociais "do mundo real" podem influenciar uma gama de mudanças comportamentais relacionadas ao tabagismo [Christakis \& Fowler 2008]. Autores referenciam que projetos baseados na web que viabilizam apoio social de pares através de fóruns, e-mails e salas de bate-papo resultaram em aumento da possibilidade de sucesso daqueles que lutam para vencer o tabagismo nos três primeiros meses em até três vezes mais [Cobb, Graham, Bock, Papandonatos \& Abrams 2005].

O presente texto introduz um sistema computacional, nomeado TratBem, projetado para acompanhar o paciente nas várias fases de mudança de hábitos, fornecendo-lhe apoio social, informacional e psicológico. A viabilização e eficácia do TratBem foi avaliada através de dois estudos realizados entre pacientes inscritos no programa de combate ao tabagismo do Hospital de Messejana Dr. Carlos Alberto Studart Gomes, aqui reportados.

\section{Teorias que Influenciaram a Concepção do TratBem}

Dentre os fatores que levam pacientes a evadirem do tratamento, identificam-se a falta de informação e os fatores emocionais [Cramer 1991]. Redes sociais de saúde (RSS) podem ajudar a resolver o problema. Pacientes membros dessas redes encontram outros pacientes com o mesmo tipo de enfermidade e compartilham informações sobre condições de saúde, sintomas e tratamentos, fornecendo, assim, suporte emocional, social e informacional aos pacientes-membros [Swan 2009]. 
Liu [Liu, Hirano, Tentori, Cheng, George, Park \& Hayes 2011] lamenta a falta de participação de profissionais de saúde nas RSS existentes. Esse engajamento é essencial, uma vez que reforça a intervenção dos conceitos: responsabilidade; credibilidade, benevolência, e experiência [Mohr, Cuijpers \& Lehman 2011]. Zbikowski et al. [Zbikowski, Hapgood, Smucker Barnwell \& McAfee 2008] constataram aumento nas taxas de sucesso quando há contato eletronicamente mediado entre profissionais e pacientes. Pulverman também corrobora essa visão quando afirma que aplicativos para smartphone voltados ao apoio à cessação tabágica incorporarão facilidades de acesso aos profissionais de saúde à medida em que precisarem [Pulverman 2014].

Entretanto, a construção de aplicativos e rede de apoio não são suficientes. É imprescindível a decisão e, principalmente, a motivação do tabagista para a mudança de hábitos. Nesse ponto, a intervenção proposta busca apoio teórico no modelo transteórico de mudança de comportamento. Construído por Prochaska, envolve teorias cognitivocomportamentais, existencial/humanista, psicanálise e gestalt/experiencial. $\mathrm{O}$ autor sugere que mudanças bem sucedidas dependem da aplicação de estratégias certas no momento adequado [Prochaska, Diclemente \& Norcross 1992]. TratBem tem a proposta de apoiar o paciente nas fases de preparação, ação e manutenção, previstas pelo modelo.

O TratBem também é fortemente influenciado pela Teoria Social Cognitiva de Bandura [Bandura 2008], principalmente nos conceitos de autoeficácia, definida como: crença de que o indivíduo tem sobre a sua capacidade de ter sucesso em uma dada atividade. Essa influência se realiza no TratBem à medida em que se faculta ao paciente uma série de definições acerca de seu processo de parada. O intuito é fortalecer no paciente o construto.

Por fim, o programa é norteado pelas visões de aprendizado adulto, propostas por Yellowlees e Marks [Yellowlees e Marks 2006]. Os autores preconizam o aprendizado: 1) just-in, quando a informação chega no momento preciso para promoção da mudança de atitude; 2) baseado em problema e; 3) altamente interativo. No TratBem, em momentos de fissura, o paciente aciona o sistema e recebe informações de como superar sua vontade de fumar. Em oposição às tradicionais cartilhas, todo processo de repasse de informações ocorre de maneira paulatina e interativa, buscando capturar a atenção do paciente, ajudando-o a construir estratégias que o levem a consolidar seu processo de mudança de hábitos.

\section{Descrição do Sistema TratBem}

A figura 1 apresenta o TratBem como elemento central no apoio ao paciente, acompanhando-o e fornecendo-lhe suporte informacional, social e psicológico, quer seja através de interações diretas com o computador, quer servindo de mediador entre paciente/profissionais de saúde ou entre pacientes/amigos ou familiares. O TratBem é dotado de chabots para garantir disponibilidade de suporte 24/7. A interação com chabots dá-se tanto por meio de troca de mensagens SMS, como por aplicativos para Smartphone (iOS, Android). 


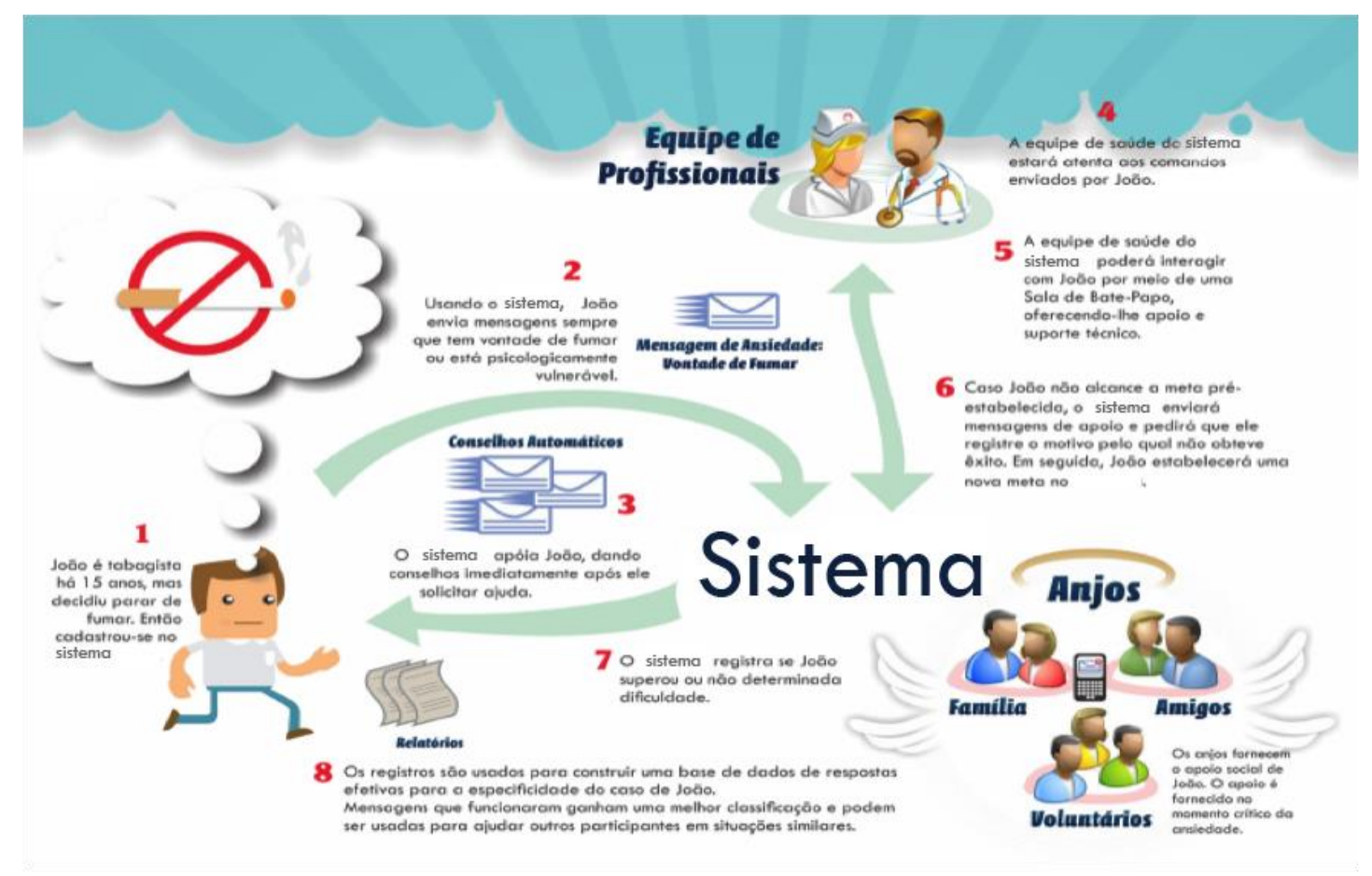

Figura 1. Descrição dos diferentes modos de interação do usuário com o TratBem.

A pervasividade do TratBem, obtida através do uso de celulares como meio primário de comunicação, confere-lhe a capacidade de, por exemplo, oferecer suporte aos pacientes nos momentos em que estão mais vulneráveis, já que o dispositivo, via de regra, acompanha seus usuários sempre que estão portando seus celulares. O suporte pode vir sob a forma de conselho, de informação contextualizada ou ainda pelo apoio dos parentes, amigos ou profissionais de saúde, que são recrutados automaticamente, através de subsistemas de notificação. A rede é persuasiva, pois objetiva persuadir o paciente a mudar de atitude frente a situações-gatilho. $\mathrm{O}$ suporte informacional e social é fornecido através da participação de profissionais de saúde e pessoas com influência e/ou convívio diário com o paciente.

Ao aderir ao TratBem, o paciente preenche um formulário eletrônico em que escolhe dentre diferentes planos, qual julga com mais condições de sucesso (autoeficácia). O sistema sugere que a escolha do dia de parada esteja pelo menos quinze dias à frente, para que o paciente passe pela fase de preparação (Modelo Transteórico). A figura 2 mostra mensagens enviadas ao paciente antes de sua parada. Já a figura 3 mostra mensagens enviadas ao paciente após a parada, configurando a fase de manutenção, também prevista no modelo de Prochaska [Prochaska et al 1992]. 


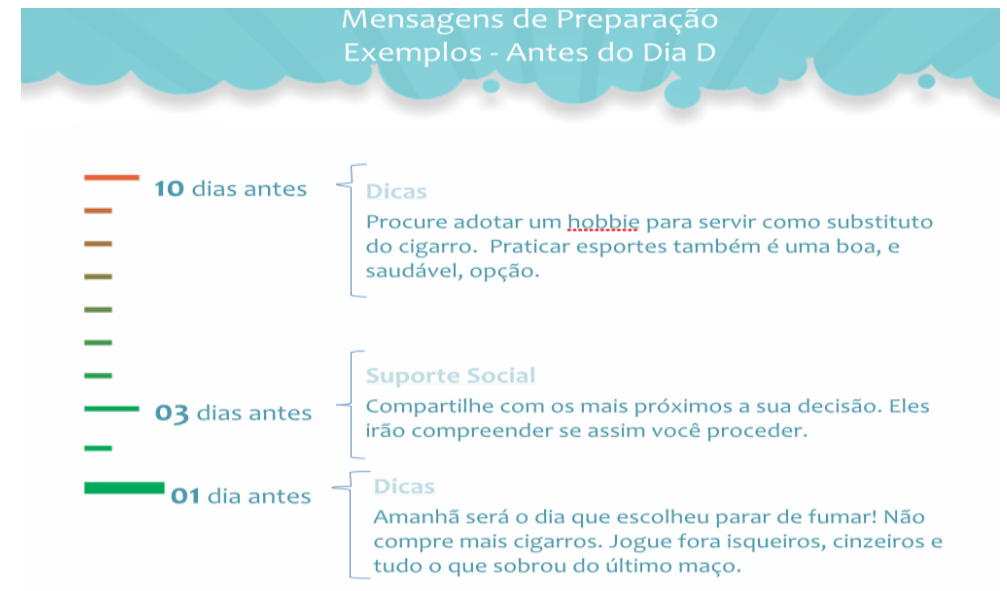

Figura 2. Interação do paciente tabagista com o sistema antes do dia agendado para sua parada.

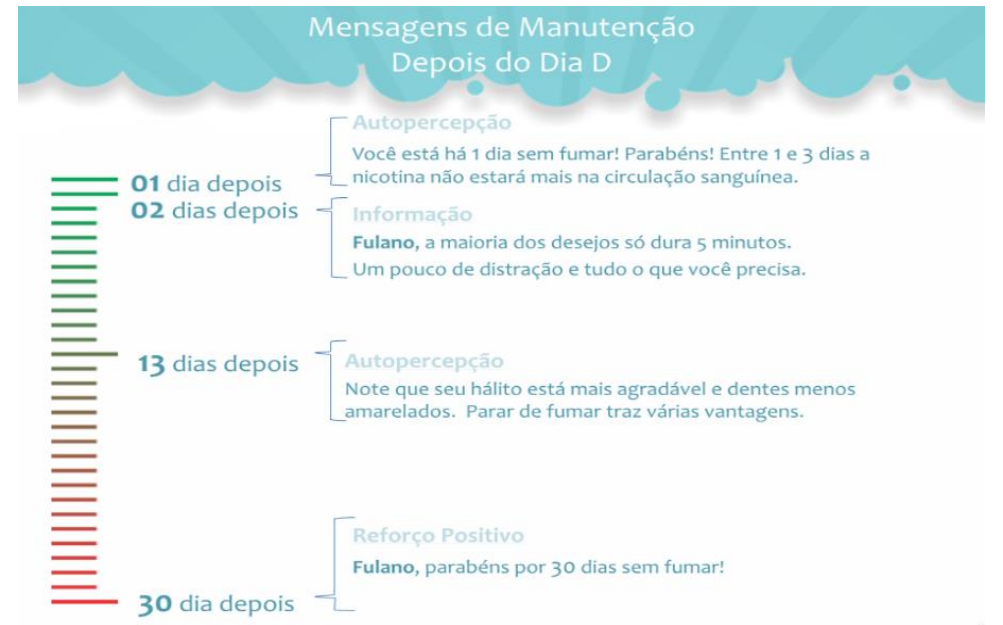

Figura 3. Interação do paciente tabagista com o sistema após o dia agendado para sua parada.

O componente social do TratBem diferencia-se sobremaneira de outras redes sociais de saúde. No modelo aqui proposto, não há interação direta entre pacientes. A interação entre eles, eletronicamente mediada, pode se constituir condutor de influências negativas, sendo, portanto, controversa e desaconselhável para o corrente estado de desenvolvimento da intervenção. O paciente pode convidar, via TratBem familiares e amigos para acompanharem todo seu processo de mudança. No convite, o paciente pode limitar o acesso de seu convidado as informações pertinentes em seu tratamento. Ele tem a possibilidade de, por exemplo, definir quem em sua rede será notificado quando tiver uma recaída.

A figura 4 ilustra como amigos e parentes são recrutados pela rede para ajudar o paciente. Vale notar que o TratBem incentiva o amigo/parente a tomar uma atitude com relação ao paciente em momentos especiais. Isso é possível, pois a rede acompanha a evolução do mesmo. A ação, no mundo real, por alguém com influência direta sobre o paciente constitui-se um importante suporte social. Membros de sua rede podem gravar mensagens de texto, áudio e vídeo que serão enviadas automaticamente sempre que determinado evento ocorre. Por exemplo, caso o paciente envie uma mensagem 
queixando-se estar triste, uma mensagem de apoio no mesmo instante será enviada a ele pelo sistema.

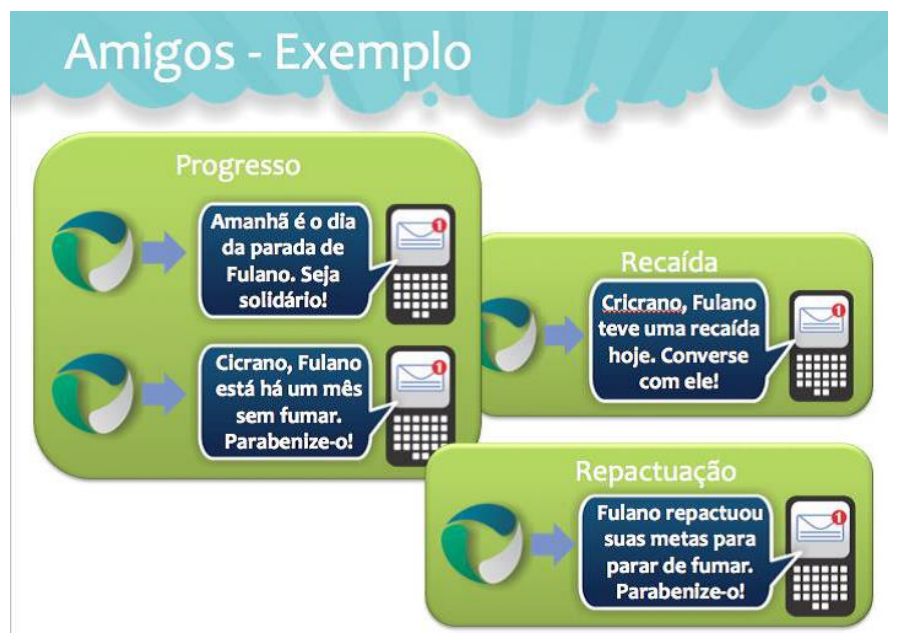

Figura 4. Mensagens enviadas pelo Sistema TratBem ao amigo, alertando sobre o processo de parada do paciente.

Finalmente, o paciente pode autorizar o TratBem a fazer publicações automáticas no Facebook e Twitter. Esse processo é orientado a eventos, como "trigésimo dia sem fumar", por exemplo.

Pacientes acessam seu painel de controle (PC) no sítio da rede na Internet. Esse recurso foi concebido para que ele consiga monitorar seu progresso, planejar como irá mudar de hábitos, convidar pessoas para fazer parte de sua rede, etc. É no PC que repactua suas metas sempre que tiver uma recaída e precisar recomeçar o processo. O PC implementa os conceitos de auto eficácia [Bandura 1986].

Dado um diálogo entre um paciente e o TratBem, há a possibilidade de somente o sistema responder às mensagens do paciente, porém profissionais de saúde são notificados sempre que pacientes iniciam uma interação e ficam monitorando a conversação, podendo entrar no chat caso julguem necessário. Membros da rede, autorizados pelo paciente, também são notificados e podem entrar no chat. Ao detectar a presença de um humano no suporte ao paciente, o sistema se retrai para posição de "ouvinte" e, quando detecta "silêncio", intervém perguntando se o atendimento foi satisfatório, ou não. A resposta do paciente à pergunta determina o fim de um "atendimento".

\section{Primeiro Estudo: Viabilidade do Uso de Ferramentas em Pacientes do Sistema Único de Saúde (SUS)}

O alcance é uma das principais justificativas para construção da intervenção. Pacientes do SUS, que geralmente possuem menor acesso aos serviços privados de saúde, compõem um dos grupos que mais se beneficiou com a utilização do Sistema. Entretanto, surgiram questões relativas à viabilidade do emprego de computação móvel dentre os beneficiários do SUS. O Hospital de Messejana possui diversos grupos de combate ao tabagismo e, naturalmente, tornou-se o campo de pesquisa adotado. 
O TratBem foi apresentado a participantes desses grupos em palestra. Em seguida, foram entrevistadas 20 pessoas (16 mulheres e 4 homens, de 37 a 68 anos). Todas eram alfabetizadas e possuíam celular. Desses, $90 \%$ deixavam o celular sempre ao alcance, mesmo quando estão em casa ou no trabalho, sendo que somente $15 \%$ possuíam smartphones. Dentre a amosta, 95\% liam as mensagens SMS que recebem, $50 \%$ enviavam mensagens via SMS rotineiramente, e $80 \%$ possueíam internet em casa. Apenas 5\% acreditavam que o TratBem não os ajudaria na luta contra o cigarro, $80 \%$ estavam certos que seriam beneficiados. Quanto à participação na rede, $80 \%$ convidariam seus (suas) companheiros(as) a fazer parte de sua rede social; $75 \%$ convocariam os amigos, $75 \%$ apresentaria o TratBem a amigos fumantes; $80 \%$ pediriam a companheiros(as) que gravassem mensagens de apoio; $75 \%$ pediriam apoio a seus amigos fumantes ou não; $76 \%$ acreditavam que o envolvimento de familiares os beneficiaria, enquanto $70 \%$ entenderam que o envolvimento de amigos poderia ser benéfico. Por fim, $72 \%$ dos entrevistados enviariam mensagens (pedindo ajuda) ao TratBem quando estivessem em fissura; $76 \%$ permitiriam que o TratBem notificasse algum membro de sua rede social quando estivessem em fissura; $78 \%$ permitiriam que o TratBem notificasse algum membro de sua rede social acerca de seus progressos.

Os resultados mostram que os pacientes possuíam acesso aos meios de comunicação utilizados pela rede, sabiam como utilizá-los, entendiam a importância dos mecanismos de apoio disponibilizados e se declararam ansiosos por usá-los. Entretanto, vê-se que o percentual de usuários com smartphone ainda é baixo dentre usuários do SUS, o que limita o alcance do TratBem nesta população. Ressalta-se, entretanto, que existe uma forte tendência de reversão desse quadro [Marinatto 2013].

\section{Segundo Estudo: Avaliação da Eficácia do TratBem em Pacientes SUS}

TratBem ficou, por um ano, disponível para livre e gratuita adesão da população brasileira, com mínima divulgação (página do TratBem no Facebook e palestras no Hospital de Messejana). Durante esse período, centenas de pessoas aderiram ao programa. Hoje o programa continua gratuito apenas para grupos de pesquisa. Nessa seção, será apresentada a forma como o TratBem impactou a vida dessas pessoas.

\subsection{Metodologia}

Pacientes que estavam há pelo menos um mês sem fumar, de acordo com o sistema, foram selecionados para compor a amostra. Dentre eles, havia integrantes do programa de cessação tabágica do Hospital de Messejana e os que se cadastraram pela internet (sem nenhum outro tipo de apoio). Cento e trinta pacientes foram entrevistados por telefone e, para efeito de análise, os pacientes foram divididos em dois grupos. Pacientes do Hospital de Messejana que utilizaram o TratBem constituem o grupo A $(n=95)$, enquanto os pacientes que usaram somente o sistema, sem qualquer outro método de apoio, formaram o grupo $\mathrm{B}(\mathrm{n}=35)$.

Em um questionário semiestruturado perguntou-se: 1) "Você está realmente conseguindo parar de fumar?" (Sim; Não; Não, mas estou diminuindo); 2) "Você usa, ou já utilizou algum outro método além do TratBem?" (Sim, utilizo; Sim, mas não mais; Não); 3) "Se sim, quais?"; 4) "Você já interagiu com o sistema?" (Sim; Não); 5) "Se não, por quê?" ;6) "Se sim, como?" (Comandos; Respondendo os quizzes; Usando o sistema no momento de fissura; Acessando o blog; Acessando a página do Facebook); 7) "Você possui amigos cadastrados?" (Sim; Não); 8) "Se sim, já recebeu ajuda 
deles?"; 9) "Se não, por quê?"; 10) “O sistema já o ajudou a não fumar?"; e 11) "Se sim, como?"

\subsection{Análise dos Dados}

\subsection{1. Índices de Abstinência}

Concluiu-se que $63,16 \%$ do grupo A e $31,58 \%$ do grupo B pararam de fumar. Observase diferença estatística significativa entre os índices de abstinência dos pacientes do grupo A e pacientes do grupo B - Chi-Square (pearson), $C^{2}(2, N=133)=16.925, p=$ 0.0002 .

Assim, a utilização de outros métodos aliada ao uso do TratBem mostrou-se mais eficaz do que o uso do programa isoladamente. Vale ressaltar, que, historicamente, o grupo anti-tabagismo do Hospital de Messejana tem uma taxa de abstinência de cerca de 50\% [Sales et al. 2006], o que sugere uma relevância na incorporação do TratBem ao tratamento de pacientes tabagistas. Por outro lado, apenas $5 \%$ das pessoas que tentam parar de fumar por conta própria tem sucesso [Westmaas et al., 2010]. Desse modo, a taxa de $31,58 \%$, aqui reportada, é bastante promissora.

\subsubsection{Suporte Psicológico}

Infelizmente, somente 4 pacientes (3 do grupo A, 1 do grupo B) utilizaram o recurso chat, ou seja, interagiram com o TratBem em momento de fissura. Todos eles afirmaram que estavam conseguindo parar de fumar. Argumentou-se que o custo do envio de mensagens SMS inibiu o uso do recurso, já que boa parte da amostra era de baixa renda.

\subsubsection{Suporte Social}

Quanto ao suporte social, 33.33\% dos pacientes do grupo B que possuíam amigos ativos estavam abstêmios até o dia da entrevista. Esse número é maior que os $31.58 \%$ dos pacientes do grupo B, quando considerados aqueles com e sem amigos cadastrados. Não foram considerados os pacientes do grupo A, dado que eles já participavam de seções presenciais no Hospital de Messejana.

\subsubsection{Percepção Geral do TratBem}

Para 73.68\% dos pacientes, o TratBem teve papel importante na preparação, ação e manutenção de abstinência. Relevantemente, existe uma correlação entre o fato de reconhecerem o papel do TratBem no tratamento com a abstinência. Há mais abstêmios entre os que responderam "sim", 60.20\%, que dos que responderam "não", $37.14 \%$. Essa diferença é considerada estatisticamente significante, Chi-Square (pearson), $\boldsymbol{C}^{2}=$ $(2, N=133)=10.466, p<0.05$.

\section{Considerações Finais}

No presente texto, abordou-se a necessidade de disponibilização de ferramentas de maior alcance no combate ao tabagismo. O Sistema em rede TratBem foi apresentado (precedido de uma exposição teórica), um estudo de viabilidade de intervenção em saúde pública apoiada por computação móvel, seguida de análise de impacto do TratBem na vida de tabagistas. 
Observou-se que, a maioria dos pacientes possuem celular, mas não do tipo smartphone. Constatou-se também que o custo do envio de SMS por parte dos pacientes do SUS inibiu a exploração e o uso das funcionalidades interativas do programa. Apesar dessa subutilização, o TratBem mostrou-se capaz de aumentar as taxas de abstinência, tanto quando utilizado sozinho, como no apoio a pacientes engajados nos grupos do Hospital de Messejana. Entretanto, faz-se necessária a replicação desse estudo em outros grupos de tabagistas para que os índices aqui apresentados possam ser refutados ou confirmados.

\section{Referências}

Bandura, A. (1986). Social foundations of thought and action: A social cognitive theory. Universidade de Michigan. Prentice-Hal.

Bandura, A., Azzi, R. G., Polydoro, S. (2008). Teoria Social Cognitiva: conceitos básicos. Porto Alegre: Artmed, 176 pp.

Christakis, N. A., Fowler, J. H. (2008). The collective dynamics of smoking in a large social network. England. New England Journal of Medicine, 358, 2249-2258.

Cobb, N. K.; Graham, A. L.; Abrams, D. B.; et al (2010). Social network structure of a large online community for smoking cessation. American Journal of Public Health, $100,1282$.

Cobb, N. K., Graham, A. L., Bock, B. C., Papandonatos, G., and Abrams, D. B. (2005). Initial evaluation of a real-world Internet smoking cessation system. Nicotine \& Tobacco Research, 7, 207-216.

Cobb, N. K., Graham, A. L., Byron, M. J., Niaura, R. S., Abrams, D. B., et al (2011). Online social networks and smoking cessation: a scientific research agenda. Journal of Medical Internet Research.

Cramer, JA. (1991). Identifying and improving compliance patterns: A composite plan for health care. New York: Raven Press.

Liu, L.S., Hirano, S.H., Tentori, M., Cheng, K.G., George, S., Park, S.Y., Hayes, G.R. (2011) Improving communication and social support for caregivers of high-risk infants through mobile technologies. Proceedings of the 14th Conference on Computer Supported Cooperative Work. ACM, New York, pp. 475-484.

Marinatto, L. (2013). Quinto país no número de celulares, Brasil pode subir ainda mais no ranking com a desoneração de smartphones. Infoglobo Comunicações e Participação S.A. Disponível em: http://extra.globo.com/noticias/celular-etecnologia/quinto-pais-no-numero-de-celulares-brasil-pode-subir-ainda-mais-noranking-com-desoneracao-de-smartphones-8123620.html. Acessado em: $15 \mathrm{dez}$ 2014.

Ministério da Saúde (2013). Controle ao tabagismo será ampliado na rede pública de saúde. Disponível

em: http://www.brasil.gov.br/noticias/arquivos/2013/04/08/controle-ao-tabagismo-seraampliado-na-rede-publica-de-saude. Acessado em: 16 jan 2015.

Mathers, C., Stevens, G., Mascarenhas, M. (2009). Global health risks: mortality and burden of disease attributable to selected major risks. World Health Organization. 
Michael, M., Cheuvront, C. C. J. B. (1998). Health Communication on the Internet: An effective channel for health behavior change? Journal of Health Communication, North Carolina, Taylor \& Francis, 71-79.

Moino, P. A. F. (2007). Desejabilidade social da assertividade e as dimensões culturais de Hofstede no contexto universitário. Dissertação (mestrado). Departamento de Economia, Contabilidade e Administração, Universidade de Taubaté. Taubaté, SP, Brasil.

Mohr D. C., Cuijpers, P., Lehman, K. (2011). Supportive accountability: a model for providing human support to enhance adherence to eHealth interventions. $\mathrm{J}$ Med Internet Res.;13:e30. doi: 10.2196/jmir.1602.http://www.jmir.org/2011/1/e30/v13i1e30.

Swan, M. (2009). Emerging patient-driven health care models: an examination of health social networks, consumer personalized medicine and quantified self-tracking. International Journal of Environmental Research and Public Health, Feb 5:492-525. doi: 10.3390/ijerph6020492.

Pulverman, R., Yellowlees, P. M. (2014). Smart devices and a future of hybrid tobacco cessation programs. Telemedicine and e-Health 20.3: 241-245.

Prochaska, J. O., Diclemente, C. C., Norcross, J. C (1992). In search of how people change: applications to addictive behaviour. American Psychologist, Washington, v. 47, p. 1102-1114.

Sales, M. P. U. et al (2006). Ambulatório de apoio ao tabagista no Ceará: perfil dos pacientes e fatores associados ao sucesso terapêutico. J. bras. pneumol., São Paulo,v.32, n.5. Disponível em http://www.scielo.br/scielo.php?script=sci_arttext\&pid=S180637132006000500007\&lng=pt\&nrm=iso. Acessado em: 13 abr 2015.

Swan, M. Emerging patient-driven health care models: an examination of health social networks, consumer personalized medicine and quantified self-tracking. Int $\mathrm{J}$ Environ Res Public Health. 2009 Feb 5;6(2):492-525. doi: 10.3390/ijerph6020492. http://www.pubmedcentral.nih.gov/articlerender.fcgitool=pu bmed\&pubmedid=19440396.

Westmaas J. L, Bontemps-Jones, J., Bauer, J. E. (2010): Social support in smoking cessation: reconciling theory and evidence. Nicotine Tob Research,12:695-707. doi: 10.1093/ntr/ntq077.ntq077

World Health Organization. (2011b). WHO Report on the Global Tobacco Epidemic, 2011: Warning About the Dangers of Tobacco. Genebra. Disponível em http://www.who.int/tobacco/global_report/2011/en/index.html.

Zbikowski, S. M., Hapgood, J., Smucker, B. S., McAfee, T. Phone and web-based tobacco cessation treatment: real-world utilization patterns and outcomes for 11,000 tobacco users. J Med Internet Res. 2008;10:e41.

Yellowlees, P. M., Marks, S. (2006). Pedagogy and educational technologies of the future. Academic Psychiatry 30.6: 439-441. 